\title{
Thermal Conductivity of Iron at High Temperatures and Pressures
}

\author{
D. Gerlich ${ }^{* \dagger}$ and R. Jeanloz ${ }^{\dagger}$ \\ ${ }^{*}$ School of Physics and Astronomy, Raymond and Beverly Sackler Faculty of Exact Sciences, \\ Tel Aviv University, Tel Aviv 69978, Israel \\ ${ }^{\dagger}$ Department of Geology and Geophysics, University of California, Berkeley, CA 94720, USA
}

The thermal conductivity of iron, $\mathrm{k}$, under conditions of high temperatures and pressures is evaluated from the measured temperature distributions in laser-heating experiments in the diamond-anvil cell. The interpretation of the results is based on the assumption that $\mathrm{k}$ is essentially electronic, and is dominated by electron-phonon scattering, normal and Umklapp processes being involved. Reasonable agreement with $\mathrm{k}$ data for iron, deduced from shock wave measurement is found.

[iron,thermal conductivity, high temperature and pressure,shock waves]

\section{Introduction}

Data of $k$ for iron under conditions of high temperature and pressures are of paramount significance in various areas. Understanding the properties of the Earth's deep interior, including the various interactions at the boundary between the lower mantle and core, depend critically on having reasonable $\mathrm{k}$ values. ${ }^{1}$ Also, the interpretation of shock-wave data for iron hinges on having reliable $\mathrm{k}$ data. ${ }^{2}$ Such data for iron are readily available from $0 \mathrm{~K}$ to the melting point at zero pressure ${ }^{3}$ and at room temperature as a function of pressure over the range $0-1 \mathrm{GPa}^{4}$

High-pressure melting experiments in the diamond anvil cell (DAC) 5 can, in principle, provide values of $k$ at elevated temperatures and pressures. In such experiments, a hot spot is formed on the upper surface of the sample by means of laser heating. The lateral temperature profile (perpendicular to the laser beam) is measured by spectroradiometry, thus furnishing a spatial temperature distribution across the hot spot. From this distribution $\mathrm{k}$ may be deduced. ${ }^{6}$ In the present communication, the results obtained by Williams et al. ${ }^{7}$ in their investigation of the high pressure melting of iron are utilized in order to evaluate k. Similar temperature profiles were determined by Boehler, ${ }^{8}$ and once differences in the laser-focus dimensions are accounted for, yield results compatible with the analysis we present here (Manga and Jeanloz, unpublished work).

\section{Calculations}

In the typical set-up for laser-heating, ${ }^{7,8}$, a powerful laser is focused on an iron sample (powder or foil) placed inside a gasketed DAC, between two layers of ruby. The radius of the focal spot is of the order of 12 $15 \mu \mathrm{m}$, and since the skin depth of iron is of the order of $0.1 \mu \mathrm{m},{ }^{9}$ all of the laser radiation is absorbed on the upper surface of the test specimen. Also, the diamond surfaces and the inner wall of the gasket hole act essentially as infinite heat sinks at ambient temperature. ${ }^{10}$ Hence, the geometry can be modeled by a three layer cylinder, denoting the layers by $\mathrm{i} 1, \mathrm{~m}$ and $\mathrm{i} 2$ respectively.

As the above configuration entails large temperature gradients, a temperature dependence of $\mathrm{k}$ in the solu- tion of the heat flow equation must be assumed. As is well known, ${ }^{6}$ in this case the usual heat conduction (Fourier) equation applies, but with the independent variable being a normalized temperature, $\tilde{\theta}$,

$$
\tilde{\theta}=\frac{1}{k_{0}} \int_{0}^{\theta} k(\theta) d \theta
$$

where $\theta$ is the temperature above ambient, and $k_{0}$ is the thermal conductivity at ambient temperature $(\theta=0)$. The heat conduction equation for the present geometry is given as,

$$
\frac{\partial^{2} \tilde{\theta}}{\partial r^{2}}+\frac{1}{r} \frac{\partial \tilde{\theta}}{\partial r}+\frac{\partial \tilde{\theta}^{2}}{\partial z^{2}}=0
$$

The heat flux is given by $f_{L}=f_{L}^{0} \exp \left[-\left(\frac{r}{a}\right)^{2}\right]$, where $\mathrm{f}_{L}^{0}$ is the peak flux. Defining a modified temperature,

$$
\hat{\theta}=\frac{k_{0}}{f_{L}^{0}} \tilde{\theta}
$$

and obviously, $\hat{\theta}$ is also a solution of eq.(2). The boundary conditions for the problem are ambient temperature at the diamond faces and the gasket, and flux matching at the boudaries between the iron and ruby layers. Equation (2) is now solved numerically by a finite differences method, ${ }^{11}$ in each of the three regions, $\mathrm{i} 1, \mathrm{~m}$ and $\mathrm{i} 2$, taking into account the above boundary conditions. The pressure dependence of the thermal conductivity of ruby, $\mathrm{k}^{i}$, at ambient temperature, required in the above calculation, is deduced from the relation, ${ }^{12} \delta k^{i} / k^{i}=-7 \delta \rho / \rho$, where $\rho$ is the density. The changes in the density of ruby under pressure are taken from Jephcoat et al..$^{13}$ After a small number of runs $(5-10)$, the solution for $\hat{\theta}$ on the heated heated metal surface, which are the only data required for evaluating $\mathrm{k}$, becomes stable.

\section{Results}

In Fig. 1 , the values of $\hat{\theta} / \hat{\theta}_{\text {max }}$, where $\hat{\theta}_{\text {max }}$ is the maximum value of $\hat{\theta}$, are shown as a function of the lateral distance. Here, the dots are the numerical solution, while the continuous line is a Gaussian curve fit to the data points over the hot zone. As can be seen, the $r$ 
dependence of these $\hat{\theta}$ data points over the hot spot

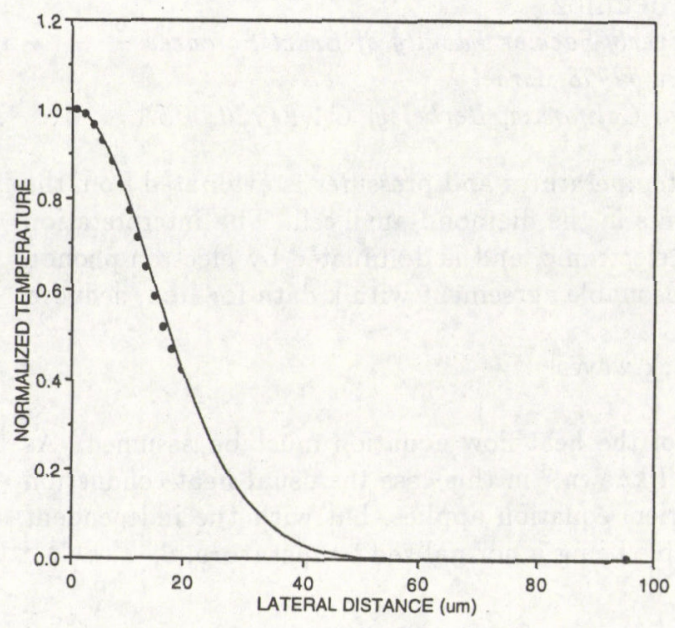

Fig. $1 \hat{\theta}$ as afunction of the lateral distance for the laser heated metal surface.

and in its close neighborhood is well represented by a Gaussian distribution, which is to be expected. ${ }^{10}$. For larger lateral distances, the decrease rate in $\hat{\theta}$ is slower than for a Gaussian curve.

From equations (1) and (3), one obtains immediately,

$$
\frac{k}{f_{L}^{0}}=\frac{\partial \hat{\theta} / \partial r}{\partial \theta / \partial r}
$$

Hence, by a numerical differentiation of the above solution, and from the numerical differentiation of the temperature distribution curves measured in iron melting experiments, ${ }^{7-8} \mathrm{k} / \mathrm{f}_{L}^{0}$ as a function of temperature for a series of pressure values may be calculated.

In order to convert $k / f_{L}^{0}$ into actual thermal conductivity data, at least one value of $k$ for a known temperature at the respective pressures is required. This is achieved, by assuming that the thermal conductivity is mainly electronic conduction, and that the dominant scattering mechanism is electron-phonon scattering. ${ }^{14}$ Also, the volume, $\mathrm{V}$, dependence of the Grüneisen constant, $\gamma$, will be assumed to have the form, ${ }^{15-17} \gamma / \gamma_{0}=V / V_{0}$, where subscript 0 refers to ambient pressure. Under these assumptions, and for high temperatures (large with respect to the Debye temperature, $\theta_{D}$ ), the volume (pressure) dependnce of $\gamma$ is readily evaluated. Thus, $\mathrm{k}$ at ambient temperature may be evaluated as a function of volume (pressure) from the known value for zero pressure. ${ }^{3} \mathrm{Al}$ though this approximation ignores the possible influence of the $\alpha-\epsilon$ transition in iron, it should be noted that the result for $\partial \ln k / \partial P$ in the $0-20 \mathrm{GPa}$ range, $2.8 \times 10^{-2} \mathrm{GPa}^{-1}$, agrees with the value measured directly ${ }^{4}$ over the range $0-1 \mathrm{GPa}, 3.5 \times 10^{-2} \mathrm{GPa}^{-1}$.
Hence the above approximation may be considered quite a reasonable. In this way, the factor $f_{L}^{0}$ may be eliminated, and absolute values for $\mathrm{k}$ can be deduced.

Thermal conductivity data can also be derived from shock wave measurements, invoking the Wiedemann-Franz relation, $k=L \sigma T$, where $\sigma$ is the electrical conductivity, $\mathrm{T}$ the absolute temperature, and $\mathrm{L}$ the Lorentz number. ${ }^{18}$ Since $\mathrm{L}$ is nearly constant for most metals and essentially independent of temperature, ${ }^{19}$ it may be safely assumed that it is also independent of pressure. Thus, from the measurements of $\sigma$ for iron under shock-waves conditions ${ }^{20,21}$ and evaluating the pressure corresponding to temperature $\mathrm{T}$ from the iron Hugoniot curve, ${ }^{22} \mathrm{k}$ may be evaluated for a series of corresponding $\mathrm{P}, \mathrm{T}$ values along the Hugoniot for iron.

In Fig. 2, $\mathrm{k}$ data obtained by various methods

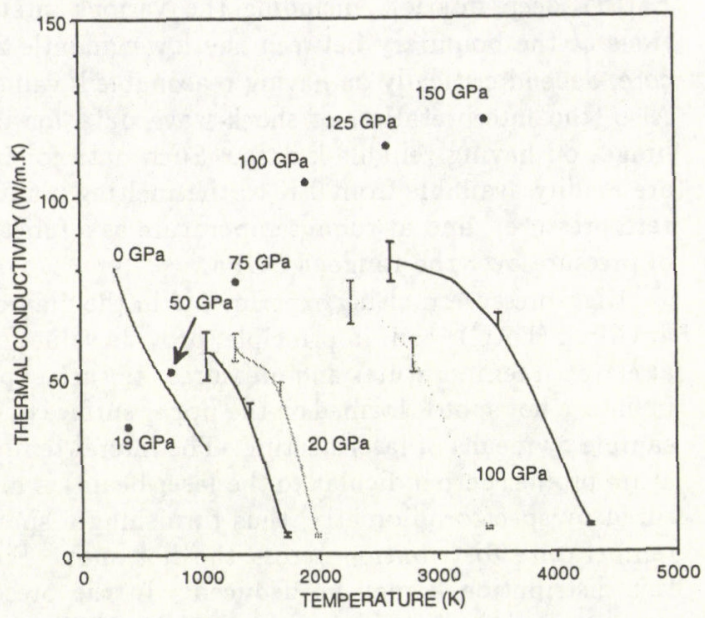

Fig. 2 Thermal conductivity as a function of pressure in the present work (curves at 0,20 and $100 \mathrm{GPa}$ ) together with similar data from shock waves.

are presented. The $0 \mathrm{GPa}$ line is derived from direct measurements, ${ }^{3}$ the 20 and $100 \mathrm{GPa}$ curves are the values obtained by the present procedure with their associated errors, and the dots are the data evaluated from shock wave measurements.

\section{Discussion}

As is well known, under the assumptions of electronic conduction dominated by normal process electronphonon scattering, the high temperature $\mathrm{k}$ should be constant. ${ }^{18,23}$ More precisely, there is a minimum around $0.2 \theta_{D}$, and a moderate rise in $\mathrm{k}$ with increasing temperature from there. If Umklapp processes are invoked as well, this rise in $\mathrm{k}$ is depressed, ${ }^{18,23}$ and the high temperature $\mathrm{k}$ is essentially constant. In the case of iron, even the direct measurements of the high temperature $\mathrm{k}$ at zero pressure show a monotonic decrease without any indications of a plateau being 
reached. This might be due to either the approach of the $\alpha-\epsilon$ transition, or a strong influence of Umklapp precesses, because of the complicated structure of the Fermi surface. This may, perhaps explain the unusual behavior of the high temperature $\mathrm{k}$ of iron. Alternatively, the scaling used to deduce the thermal conductivity of the insulating (ruby) layers, at high temperatures and pressures may need to be re-examined, thus possibly modifying the boundary conditions in the present analysis. Altering the boundary conditions would change the calculated temperature distribution, and therefore the temperature dependence for the iron sample at high pressure.

Considering the assumptions involved in fixing the vertical position of the 20 and $100 \mathrm{GPa}$ lines (eliminating the factor $\mathrm{f}_{L}^{0}$ ), and the fact that shock data at low Hugoniot pressures have large errors, the agreement between the present data and the values deduced from the shock wave measurements may be considered quite satisfactory. Thus, it is concluded that the scheme outlined in the present paper may be a useful tool in calculating thermal conductivity values as a function of pressure and temperature from melting curves.

\section{Acknowledgements}

We thank M. Manga for helpful discussions. Work supported by NASA and NSF.

\section{References}

[1 ] R. Jeanloz, Ann. Rev. Earth Planet. Sci. 18, 357 (1990).

[2 ] H. Tan and T.J. Ahrens, High Press. Res .2, 159 (1990).

[3 ] Y.S. Touloukian and E.H. Buyco (eds.), Thermophysical Properties of Matter, The TRPC Data Center, Thermal Conductivity 1, (1970).

[4] B. Sundqvist, in High Pressure in Research and Industry, C-M Backman, T. Johanisson and L. Tenger (eds.), p. 432 (Private Edition, Uppsala, 1982).

[5 ] R. Jeanloz and A. Kavner, Phil. Trans. Roy. Soc. (London), A354, 1279 (1996).

[6 ]6 H.S. Carslaw and J. Jaeger, Conduction of Heat in Solids, 2nd ed. Clarendon Press, Oxford (1986).
[7 ] Q. Williams, E. Knittle and R. Jeanloz, J. Geophys. Res. 96, 2171 (1991).

[8 ] R. Boehler, Nature 363, 534 (1993).

[9 ] D. W. Lynch and W. R. Hunter, in Handbook of Optical Constants of Solids II (E. D. Palik, ed.) pp. 341-419, Academic Press, New York (1991).

[10 ] S. Bodea and R. Jeanloz, J. Appl. Phys. 65, 4688 (1989).

[11 ] William H. Press, Brian R. Flannery, Saul A. Teukolsky and William T. Wetterling, Numerical Recipes, Cambridge University Press, Cambridge (1989).

[12 ] M. Roufosse and R. Jeanloz, J. Geophys. Res. 88, 7399 (1983).

[13 ] A.P. Jephcoat, R.J. Hemley and H.K. Mao, Physica B+C150, 115 (1988).

[14 ] L. Bohlin, Sol. State Comm. 19, 389 (1976).

[15] R. Boehler, J. Geophys. Res. B87,5501 (1982).

[16 ] W.A. Bassett, A. Takahashi, H.K. Mao and J.S. Weaver, J. Appl. Phys. 38, 319 (1968).

[17 ] C.A. Swenson, Phys. Rev. B31, 1150 (1985).

[18 ] J.M. Ziman, Electrons and Phonons, Clarendon Press, Oxford (1960):

[19 ] G. Grimvall, Thermophysical Properties of Materials, North-Holland, New York

[20 ] R.N. Keeler and A.C. Mitchell, Sol. State Comm. 7, 271 (1969).

[21 ] G. Matassov, The Electrical Conductivity of Iron-Silicon Alloys at High Pressures and the Earth's Core, Ph.D. Thesis, University of California, Davis (1977).

[22 ] R.G. McQueen, S.P. Marsh, J.W. Taylor, J.N. Fritz and W.J. Carter, in High Velocity Impact Phenomena, R. Kinslow (ed.), p. 293, Academic Press, New York (1970).

[23 ] R. Berman, Thermal Conduction in Solids, Clarendon Press, Oxford (1976). 